Received: February 21, 2017

Accepted: April 10, 2017

Published: April 20, 2017

\section{New Approaches to Melanoma Treatment: Checkpoint Inhibition with Novel Targeted Therapy}

\author{
Suzie Chen $^{1 *}$ and Ann Robinson ${ }^{2}$ \\ ${ }^{1}$ Professor, Rutgers, the State University of New Jersey, Piscataway NJ \\ ${ }^{2}$ Graduate Student, Rutgers, the State University of New Jersey, Piscataway NJ
}

*Corresponding author:Suzie Chen, Professor, Rutgers, the State University of New Jersey, 164 Frelinghuysen Road, Piscataway NJ 08854-8020, Tel: 848-445-7243; Fax: 732-445-0687; E-mail: suziec@pharmacy.rutgers.edu

\section{Abstract}

Melanoma is the most dangerous type of skin cancer, largely due to its propensity for recurrence and metastasis, even after removal of malignant tissue. When melanoma reaches advanced stages, the disease becomes refractory to many types of therapy, which has created a need for novel therapeutic strategies to combat the disease. Our group focuses on the oncogenic function of a neuronal receptor, metabotropic glutamate receptor 1 (mGluR1). When mGluR1 is aberrantly expressed in melanocytes, elevated levels of extracellular glutamate mediate the constitutive activation of the receptor to promote cell proliferation. We are exploring the potential synergistic efficacy when combining a glutamatergic signaling inhibitor with a checkpoint inhibitor antibody.

Melanoma, a type of skin cancer arising from the uncontrolled growth of the pigmented cells of the epidermis, is the most dangerous form of skin cancer. This year, the American Cancer Society expects over 85,000 new cases of melanoma to be diagnosed and about 10,000 people to die from the disease. Skin abnormalities, such as moles or freckles, are common, melanoma, though, is likely to undergo changes over time including variations to color, size, or symmetry of a skin lesion. Over the past three decades, the number of melanoma cases in the United States has continued to increase. Health professionals have advised the public that if concern arises regarding a new or evolving nevus, visiting a dermatologist can help determine whether treatment is necessary [1].

When melanoma is diagnosed at its earliest stages, surgical resection of the malignant tissue is the most common treatment and often results in patients surviving melanoma-free for many years after the initial diagnosis. However, compared to other types of cancer, melanoma has a high incidence of recurrence. Another dangerous characteristic associated with re-emergence of melanoma is the propensity to metastasize to vital organs, particularly the brain and lungs. Once melanoma recurs or metastasizes, the disease becomes much more difficult to treat. At this stage, surgery is no longer a viable treatment option,

therefore patients may undergo various forms of chemotherapeutic and/or radiation therapies [2].

Whole Brain Radiation Therapy (WBRT) is commonly used in patients with brain metastases, however, patient survival rate was not drastically improved, as compared to patients who received only palliative care [3]. Additionally, WBRT has been shown to negatively impact quality of life for some patients receiving this treatment as an adjuvant to other therapies [3,4]. Because of these drawbacks, WBRT has been limited to use in patients with widespread or large volume brain metastases.

One radiation treatment alternative to WBRT, is Stereotactic Radiosurgery (SRS), also known as CyberKnife or Gamma Knife treatment. This modality delivers high-dose radiation by using multiple radiation beams that converge at a defined point where the tumor is located. Utilizing radiation beams in this grid-like manner allows delivery to a specific region, while preventing high doses of radiation exposure to adjacent, noncancerous areas [2]. Delivery systems permit treatment of multiple metastases, however, only recently have the data emerged supporting its use for brain metastases [2,5]. Recent studies have shown comparable improvements to survival in groups with a single secondary tumor versus groups with as many as ten secondary tumors, however, melanoma-specific responses to this therapy have yet to be investigated $[2,5]$.

Another risk of melanoma is high resistance to traditional therapies like radiation. To combat this problem, the focus of melanoma treatment has shifted towards therapies targeting specific mutations identified as "drivers" of disease. Many cases of melanoma can be attributed to aberrant activation of signaling cascades responsible for regulating cell growth, like the MitogenActivated Protein Kinase (MAPK) pathway. Activating mutations in components of this signaling cascade promote deregulated cell proliferation and suppression of apoptosis. Sorafenib, a multi- 
kinase inhibitor, has been used to target multiple steps in the MAPK pathway and is FDA approved for treating several cancers including kidney and liver [6]. Vemurafenib and dabrafenib are both used to treat melanoma patients with a point mutation substituting glutamic acid for valine at amino acid reside 600 within the BRAF gene, the most common mutation associated with melanoma. Vemurafenib was the first oral targeted mutant BRAF inhibitor approved by the FDA to treat patie-nts with unresectable or metastatic melanoma expressing mutated BRAF [7]. Metastatic melanoma patients experienced increased overall survival when treated with vemurafenib versus dacarbazine, a cytotoxic chemotherapeutic agent, and $37 \%$ of patients saw regression of intracranial tumors [8, 9]. Dabrafenib received FDA approval shortly after vemurafenib and achieved improved progression-free survival and anti-tumor activity for intracranial lesions in the $30-40 \%$ range [2].

Despite initial success with targeted therapies, many patients experience disease recurrence or stop responding to treatment. This high recurrence rate suggests melanoma can circumvent signaling blockades resulting from targeted therapy by inducing new, novel mutations, which allow the disease to progress. For this reason, identifying alternative treatment targets for melanoma remains critical.

Our lab was the first to focus on the role of a normal neuronal receptor, metabotropic glutamate receptor 1 (gene: GRM1, human; Grm1, mouse; protein: mGluR1) in melanoma development and progression. A transgenic mouse model, TG-3, developed pigmented lesions on the skin surface with progression to distant organs, including lymph nodes, brain, and lungs. Histological analysis confirmed the lesions as melanoma, while molecular gene mapping identified an insertion of several copies of the transgene, clone $\mathrm{B}$, into intron 3 of the gene encoding mGluR1. Subsequent protein and RNA assessment studies showed that mGluR1 expression was detected in tumor-bearing ears, but not in noncancerous ear tissue. We hypothesize that ectopic expression of a neuronal receptor, mGluR1, in melanocytes, a cell type where mGluR1 expression is not endogenous, results in tumor development. To test our hypothesis on the causative role of Grm1 in melanoma development, we generated another transgenic mouse line featuring Grm1 cDNA under the control of a melanocyte-specific dopachrome tautomerase promoter. As this transgenic model developed melanoma lesions very similar to the TG-3 model, we conclude that ectopic Grm1 expression alone is sufficient to induce spontaneous metastatic melanocytic neoplasia in vivo [10]. Further investigation identified mGluR1 expression in over $80 \%$ of human melanoma cell lines and $60 \%$ of human melanoma biopsies [11].

To elucidate how mGluR1 expression affects downstream signaling pathways, our lab transfected exogenous mouse Grm1 cDNA into immortalized, nontumorigenic, mGluR1-negative mouse melanocytes made from C57BL/6, melanA. Several stable Mass clones were isolated and were shown to be very tumorigenic in both immune-competent syngeneic C57BL/6 and immune-compromised nude mice. We observed robust tumor formation, angiogenesis, and invasiveness to lung and muscle tissue. Functionality assays showed functional and sustained Grm1 ex- pression, which was required to maintain the tumorigenic phenotype and establish autocrine loops with enhanced levels of extracellularglutamate, the natural ligand of mGluR1, for constitutive activation of the receptor $[12,13]$. Signaling through mGluR1 results in the generation of second messengers, inositol triphosphate (IP3) and diacyl glycerol (DAG). IP3 causes the release of calcium from the endoplasmic reticulum and DAG stimulates protein kinase $\mathrm{C}$ (PKC) which activates the MAPK and PI3K/AKT signaling cascades to promote cellular proliferation and inhibit apoptosis [12]. Once the connection between mGluR1 and downstream pathways promoting cell proliferation was established, we investigated compounds capable of suppressing or reversing this effect. The cell membrane localization of mGluR1 makes it an attractive therapeutic target [11]. Our lab, with clinician collaborators, investigated Riluzole, an FDA-approved therapy for Amyotrophic Lateral Sclerosis (ALS), as a potential inhibitor for glutamatergic signaling in preclinical studies. Riluzole functions by inhibiting glutamate release from neurons and noncompetitively blocking its uptake by post-synaptic neurons, therefore preventing neuronal glutamate toxicity [14]. Our group showed that Riluzole could disrupt glutamatergic signaling in tumor cells by disrupting glutamate release to the extracellular environment of mGluR1 expressing melanoma cells, limiting the availability of the ligand to maintain receptor activation [15]. One consequence of reduced extracellular glutamate is a decrease in cell growth, as has been shown for many transformed cells including melanoma [16]. This preclinical work has been translated to the clinic in the form of phase 0 and phase II trials for patients with advanced melanoma [17, 18]. Riluzole administration to these patients resulted in suppression of MAPK and PI3K/AKT signaling and tumor involution for 34\% of patients, independent of BRAF/NRAS mutational status, however, inhibition of mGluR1 signal transduction alone failed to change clinical outcomes in patients with advanced melanoma. Currently, we are exploring therapeutic options using Riluzole in combination with other targeted therapies like vemurafenib and rapamycin, radiation therapy, and novel immunotherapies [19, 20].

Immunotherapy seeks to activate or enhance a patient's immune response against tumor-specific antigens, allowing the immune system to target cancer cells specifically [21]. Melanoma has received special attention regarding immunotherapy because melanoma is considered to be one of the most immunogenic types of cancer. As early as the 1970s, researchers reported spontaneous regression of primary and metastatic melanoma. This observation led researchers to conclude that melanoma can be recognized and sufficiently destroyed by the immune system [22] Decades later, the promise of novel immunotherapy drugs hopes to promote disease regression in a large proportion of patients.

One of the earliest immunotherapies used to treat melanoma was administration of high-dose interleukin-2 (HD IL-2), a cytokine promoting T-cell proliferation, in high doses [23]. About $16 \%$ of melanoma patients enrolled in a clinical trial showed durable responses to HD IL-2 treatment [24]. Similar results were observed in a renal cell carcinoma clinical trial, which led to FDA approval of HD IL-2 treatment for both immunogenic diseases. The promising results of clinical trials were eventually 
overshadowed by the high toxicity in many patients. Lower doses of IL-2 showed fewer adverse effects, but were inferior to HD IL-2 treatment in generating durable anti-tumor responses [25].

Novel immunotherapies seek to enhance an immune response from cytotoxic T-cells in cancers where T-cell activity has been reduced or shut down. To prevent cytotoxic T-cells from destroying normal cells within our bodies, many cells express surface proteins allowing interaction with T-cells. When activated T-cells encounter somatic cells expressing these proteins, an inhibitory signal is generated within the T-cell, suppressing its cytotoxic effects. This interaction, called an immune checkpoint, protects us from autoimmune responses and allows for peripheral tolerance. Recent studies show that some cancer types, like melanoma, also express these immune checkpoint proteins, allowing them to evade immune responses [26]. Drugs like ipilimumab and pembrolizumab/nivolumab seek to block this interaction by inhibiting the protein receptors CTLA-4 and PD-1, respectively. Ipilimumab has been shown to improve median overall survival up to 10 months for melanoma patients. Melanoma patients treated with pembrolizumab in clinical trials showed a $26 \%$ tumor response rate, while nivolumab treatment gave $32 \%$ objective response rate with durable responses lasting longer than six months in about a third of patients who initially responded to therapy. These findings led to FDA approval of all three drugs to treat patients with advanced stages of melanoma, particularly patients who had stopped responding to targeted therapies. Some patients eventually stop responding, notably those treated with ipilimumab alone, leading researchers to explore therapies utilizing immunotherapy drugs in combination with radiation or targeted therapies [19]. The goal is to identify treatments applicable to disease in a greater proportion of patients, while also limiting disease recurrence or resistance to monotherapies.

Current work in our lab seeks to combine our knowledge of melanoma animal models and the efficacy of Riluzole treatment with information available about PD-1 inhibitors to further investigate treatments for advanced melanoma. Recent analysis of two independent cohorts revealed patients with melanoma characterized by a high mutational burden showed improved, durable responses and progression-free survival after undergoing PD-1 inhibition treatment [27]. Previous work in our lab identified substantial levels of $\gamma \mathrm{H} 2 \mathrm{AX}$, a phosphorylated histone variant associated with DNA double-stranded breaks, in Riluzole treated cell lines [19]. By inducing high levels of double-stranded breaks, Riluzole treatment may help create a high mutational burden in melanoma lesions, sensitizing cells to concurrent treatment with PD-1 inhibitors.

To explore this question, we subcutaneously inoculated the tumorigenic Mass cells generated in our lab into both flanks of C57BL/6 mice and waited for tumors to become palpable before initiating treatment. Our monotherapy treatment groups include Riluzole, and anti-PD-1 treatment, while our combination therapy group includes both Riluzole and anti-PD-1 inhibitor treatments. We hope to observe significant changes in tumor volume between monotherapy and combination therapy groups. By using checkpoint inhibitors with targeted therapy, we hope to identify treatment regimens capable of achieving anti-tumor responses without demonstrable toxicities in animal models for metastatic melanoma. Our goal is to generate preclinical data supporting the use of combination treatments in clinical trials for patients with advanced melanoma.

\section{References}

1. American Cancer Society. Melanoma Skin Cancer. 2017.

2. Jang S, Atkins MB. Treatment of melanoma CNS metastases. Cancer Treatment and Research. 2016;167:263-279. doi: 10.1007/978-3-319-22539_11

3. Fife KM, Colman MH, Stevens GN, Firth IC, Moon D, Shannon $\mathrm{KF}$, et al. Determinants of outcome in melanoma patients with cerebral metastases. J Clin Oncol. 2004;22(7):1293-1300.

4. Soffietti R, Kocher M, Abacioglu UM, Villa S, Fauchon F, Baumert BG, et al. A European Organisation for Research and Treatment of Cancer phase III trial of adjuvant whole-brain radiotherapy versus observation in patients with one to three brain metastases from solid tumors after surgical resection or radio surgery: quality-of-life results. J Clin Oncol. 2013;31(1):65-72. doi: 10.1200/JCO.2011.41.0639

5. Yamamoto M, Serizawa T, Shuto T, Akabane A, Higuchi Y, Kawagishi J, et al. Stereotactic radiosurgery for patients with multiple brain metastases: a multi-institutional prospective observational study. Lancet Oncol. 2014;15(4):387-395.doi: 10.1016/S1470-2045(14)70061-0

6. Xia Y, Li Y, Westover KD, Sun J, Chen H, Zhang J, et al. Inhibition of cell proliferation in an NRAS mutant melanoma cell line by combining sorafenib and alpha-mangostin. PLos One. 2016;11(5). doi: 10.1371/journal.pone.0155217

7. Davies H, Bignell GR, Cox C, Stephens P, Edkins S, Clegg S, et al. Mutations of the BRAF gene in human cancer. Nature. 2002;417(6892):949-954.

8. Chapman PB, Hauschild A, Robert C, Haanen JB, Ascierto $\mathrm{P}$, Larkin $\mathrm{J}$, et al. Improved survival with vemurafenib in melanoma with BRAF V600E mutation. N Engl J Med. 2011;364(26):2507-2516. doi:10.1056/NEJMoa1103782

9. Dummer R, Goldinger SM, Turtschi CP, Eggmann NB, Michielin O, Mitchell L, et al. Vemurafenib in patients with BRAF(V600) mutation-positive melanoma with symptomatic brain metastases: final results of an open-label pilot study. Eur J Cancer. 2014;50(3):611-621. doi:10.1016/j.ejca.2013.11.002

10. Pollock PM, Cohen-Solal K, Sood R, Namkoong J, Martino JJ, Koganti A, et al. Melanoma mouse model implicates metabotropic glutamate signaling in melanocytic neoplasia. Nat Genet. 2003;34(1):108-112. 
11. Teh, Chen SC. Glutamatergic signaling in cellular transformation. Pigment Cell Melanoma Res. 2012;25(3):331-342. doi: 10.1111/j.1755-148X.2012.00983.x.

12. Yu LJ, Wall BA, Chen S. The current management of brain metastasis in melanoma: a focus on riluzole. Expert Rev Neurother. 2015;15(7):779-792. doi: 10.1586/14737175.2015.1055321.

13. Shin S, Namkoong J, Wall BA, Gleason R, Lee HJ, Chen S. Oncogenic activities of metabotropic glutamate receptor 1 (Grm1) in melanocyte transformation. Pigment Cell Melanoma Res. 2008;21(3):368-378. doi: 10.1111/j.1755148X.2008.00452.x.

14. Doble A. The pharmacology and mechanism of action of riluzole. Neurology. 1996;47(6 Suppl 4):S233-S241.

15. Namkoong J, Shin SS, Le HJ, Marin YE, Wall BA, Goydos JS, et al. Metabotropic glutamate receptor 1 and glutamate signaling in human melanoma. Cancer Res. 2007;67(5):2298-2305.

16. Gelb T, Hathaway HA, Wroblewski JT. Triple threat treatment: Exploiting the dependence receptor properties of metabotropic glutamate receptor 1 against melanoma. Mol Cell Oncol. 2014;1(4):e969163. doi: 10.4161/23723548.2014.969163.

17. Mehnert JM, Lee JH, Shirk J, et al. A phase II trial of riluzole, an antagonist of metabotropic glutamate receptor 1 (GRM1) signaling in metastatic melanoma. J Clin Oncology. 2010;28(15).

18. Yip D, Le MN, Chan JL, Lee JH, Mehnert JA, Yudd A, et al. A phase 0 trial of riluzole in patients with resectable stage III and IV melanoma. Clin Cancer Res. 2009;15(11):3896-3902. doi: 10.1158/1078-0432.CCR-08-330.

19. Wall BA, Lumeng JY, Khan A, Haffty B, Goydos JS, Chen S. Riluzole is a radio-sensitizing agent in an in vivo model of brain metastasis derived from GRM1 expressing human melanoma cells. Pigment Cell Melanoma Res. 2014;28(1):105-109. doi: 10.1111/pcmr.12327

20. Rosenberg SA, Niglio SA, Salehomourn N, Chan JL, Jeong BS, Wen Y, et al. Targeting glutamatergic singaling and the PI3 kinase pathway to halt melanoma progression. Transl Oncol. 2015;8(1):1-9. doi: 10.1016/j.tranon.2014.11.001.

21. National Cancer Institute. Melanoma Treatment (PDQ): Patient Version. 2017.

22. ulkley GB, Cohen MH, Banks PM, Char DH, Ketcham AS. Long-term spontaneous regression of malignant melanoma with visceral metastases. Report of a case with immunologic profile. Cancer. 1975;36:485-494.

23. Rosenberg SA, Lotze MT, Muul LM, Leitman S, Chang AE, Ettinghausen SE, et al. Observations on the systemic administration of autologous lymphokine-activated killer cells and recombinant interleukin-2 to patients with metastatic cancer. N Engl J Med. 1985;313(23):1485-1492.

24. Atkins MB, Lotze MT, Dutcher JP, Fisher RI, Weiss G, Margolin $\mathrm{K}$, et al. High-dose recombinant interleukin 2 therapy for patients with metastatic melanoma: analysis of 270 patients treated between 1985 and 1993. J Clin Oncol. 1999;17(7):2105-2116.

25. Amin A, White RL. Interleukin-2: is it still indicated for melanoma and RCC in an era of targeted therapies? Oncology. 2013;27(7):680-691

26. Okazaki T, Honjo T. PD-1 and PD-1 ligands: From discovery to clinical application. Int Immunol. 2007;19(7):813-824.

27. Rizvi NA, Hellmann MD, Snyder A, Pia Kvistborg, Vladimir Makarov, Jonathan J Havel, et al. Cancer immunology. Mutational landscape determines sensitivity to PD-1 blockade in non-small cell lung cancer. Science. 2015;348(6230):124-128. doi: 10.1126/science.aaa1348 